Groups Geom. Dyn. 8 (2014), 311-329

DOI $10.4171 / \mathrm{GGD} / 227$
Groups, Geometry, and Dynamics

(C) European Mathematical Society

\title{
On the growth of Betti numbers in $p$-adic analytic towers
}

\author{
Nicolas Bergeron, Peter Linnell, Wolfgang Lück and Roman Sauer
}

\begin{abstract}
We study the asymptotic growth of Betti numbers in tower of finite covers and provide simple proofs of approximation results, which were previously obtained by Calegari and Emerton, in the generality of arbitrary $p$-adic analytic towers of covers. Further, we also obtain partial results about arbitrary pro- $p$ towers.
\end{abstract}

Mathematics Subject Classification (2010). 58G12, 55P99.

Keywords. Asymptotic growth of Betti numbers, $p$-adic analytic groups.

\section{Introduction and statement of results}

This paper is mainly concerned with the asymptotic growth of Betti numbers in a tower of finite covers of a compact space $X$ associated to a chain of subgroups of the fundamental group of $X$ which gives rise to a $p$-adic analytic group. Both Betti numbers with coefficients in $\mathbb{Q}$ and $\mathbb{F}_{p}$ are considered. Especially the case of $\mathbb{F}_{p^{-}}$ coefficients received a lot of attention in recent years. We only name here the work of Calegari-Emerton [5], [6], which is motivated by the $p$-adic Langlands program, and the work of Lackenby [21], [22] in group theory, which is connected to property $\tau$ and 3-manifold theory.

1.1. Global setup. With the exception of Section 4, we retain the following setup throughout this paper. Let $X$ be a connected compact CW-complex with fundamental group $\Gamma$. Let $p$ be a prime, let $n$ be a positive integer, and let $\phi: \Gamma \rightarrow \mathrm{GL}_{n}\left(\mathbb{Z}_{p}\right)$ be a homomorphism. The closure of the image of $\phi$, which is denoted by $G$, is a $p$-adic analytic group admitting an exhausting filtration by open normal subgroups:

$$
G_{i}=\operatorname{ker}\left(G \rightarrow \mathrm{GL}_{n}\left(\mathbb{Z} / p^{i} \mathbb{Z}\right)\right)
$$

Set $\Gamma_{i}=\phi^{-1}\left(G_{i}\right)$, and let $X_{i}$ be the corresponding finite cover of $X$. Let $\bar{X}$ be the cover of $X$ corresponding to the kernel of $\phi$ and $\bar{\Gamma}=\Gamma / \operatorname{Ker}(\phi)$; note that $\bar{\Gamma}$ acts properly and freely on $\bar{X}$ with quotient $\bar{\Gamma} \backslash \bar{X}=X$. Our main concern is the growth 
of the Betti numbers

$$
\begin{aligned}
b_{k}\left(X_{i}\right) & =\operatorname{dim}_{\mathbb{Q}} H_{k}\left(X_{i}, \mathbb{Q}\right) ; \\
b_{k}\left(X_{i}, \mathbb{F}_{p}\right) & =\operatorname{dim}_{\mathbb{F}_{p}} H_{k}\left(X_{i}, \mathbb{F}_{p}\right),
\end{aligned}
$$

with coefficients in $\mathbb{Q}$ and $\mathbb{F}_{p}$ as functions of $i$.

1.2. Growth of Betti numbers in a $p$-adic analytic tower. W. Lück proved that for each integer $k$ the sequence $b_{k}\left(X_{i}\right) /\left[\Gamma: \Gamma_{i}\right]$ always converges as $i \rightarrow \infty$, and the limit equals the $k$-th $L^{2}$-Betti number $\beta_{k}(\bar{X}, \bar{\Gamma})$ of the action of $\bar{\Gamma}$ on $\bar{X}$. In that context we obtain the following result on the rate of convergence in terms of the dimension of $G$ as a $p$-adic analytic group. We refer to [12], Theorem 8.36 on p. 201, for equivalent characterizations of the dimension of $G$.

Theorem 1.1. Let $d=\operatorname{dim}(G)$. Then, for any integer $k$ and as $i$ tends to infinity, we have

$$
b_{k}\left(X_{i}\right)=\beta_{k}(\bar{X}, \bar{\Gamma})\left[\Gamma: \Gamma_{i}\right]+O\left(\left[\Gamma: \Gamma_{i}\right]^{1-1 / d}\right) .
$$

The novelty of Theorem 1.1 is obviously the error term. For more general covers, this has already been studied by Sarnak and Xue [28] and by Clair and Whyte [8] but they obtain much weaker results, in particular their results do not apply when 0 occurs in the $L^{2}$-spectrum of $\bar{X}$. $^{1}$

Theorem 1.1 generalizes in the case of trivial coefficients the main theorem of Calegari-Emerton [5] which deals with arithmetic locally symmetric spaces. After this paper had been put on the ArXiv Frank Calegari informed us that Theorem $1.1 \mathrm{can}$ be deduced from the method of [5]. In fact both proofs rest on a theorem of Harris, see Theorem 2.1 below, but we believe that our method of proof is somewhat simpler. We refer to Section 3 for more details on the relation to the work of Calegari-Emerton.

1.3. Growth of $\mathbb{F}_{\boldsymbol{p}}$-Betti numbers in a $p$-adic analytic tower. Homological algebra over Iwasawa algebras and the theory of $p$-adic analytic groups provide important tools to study the asymptotic growth of Betti numbers in a $p$-adic analytic tower of covers. Whilst Iwasawa algebras are hidden in the proof of Theorem 1.1, they are essential even in the formulation of a corresponding result for $\mathbb{F}_{p}$-Betti numbers. The Iwasawa algebra of $G$ over $R=\mathbb{F}_{p}$ or $\mathbb{Z}_{p}$ is the completion of the group algebra $R[G]$ :

$$
R[[G]]=\lim _{\longleftarrow} R\left[G / G_{i}\right] .
$$

The Iwasawa algebra is a right and left Noetherian domain. Further, if $G$ is torsionfree, then $R[[G]]$ does not contain zero divisors and its non-zero elements satisfy the Ore condition, see $\S 6$ of [16]. This means that the ring of fractions $Q(R[[G]])$ is a skew field, the Ore localization of $R[[G]]$. Hence there is a notion of rank:

\footnotetext{
${ }^{1}$ We should note however that in the special case of lattices in $\operatorname{SU}(2,1)$ Sarnak and Xue produce a better exponent of $\frac{7}{12}$ in the error term for the first Betti number.
} 
Definition 1.2. If $G$ is torsion-free, we define the rank of a left $R[[G]]$-module $M$ as

$$
\operatorname{rank}_{R[[G]]}(M)=\operatorname{dim}_{Q(R[[G]])}\left(Q(R[[G]]) \otimes_{R[[G]]} M\right) .
$$

For general $G$ we define the rank of $M$ as

$$
\operatorname{rank}_{R[[G]]}(M)=\frac{1}{\left[G: G_{0}\right]} \operatorname{rank}_{R\left[\left[G_{0}\right]\right]}(M),
$$

where $G_{0}<G$ is any uniform, hence torsion-free, subgroup, and $M$ is regarded as an $R\left[\left[G_{0}\right]\right]$-module by restriction.

Using the above rank, we define an analog of $L^{2}$-Betti numbers in characteristic $p$. For a CW-complex $Y$ the cellular chain complex will always be denoted by $C_{*}(Y)$. It is a consequence of the proof of Theorem 1.1 (see (2.10)) that, if you replace $\mathbb{F}_{p}$ by $\mathbb{Z}_{p}$ in the definition below, you obtain the $L^{2}$-Betti numbers of $\bar{X}$.

Definition 1.3. The mod $p L^{2}$-Betti numbers of the $\bar{\Gamma}$-space $\bar{X}$ are defined as

$$
\beta_{k}\left(\bar{X}, \bar{\Gamma} ; \mathbb{F}_{p}\right)=\operatorname{rank}_{\mathbb{F}_{p}[[G]]}\left(H_{k}\left(\mathbb{F}_{p}[[G]] \otimes_{\mathbb{F}_{p}[\bar{\Gamma}]} C_{*}\left(\bar{X}, \mathbb{F}_{p}\right)\right)\right),
$$

where $\mathbb{F}_{p}[[G]]$ is regarded as a right $\mathbb{F}_{p}[[\bar{\Gamma}]]$-module via $\phi: \Gamma \rightarrow G$.

For these characteristic $p$ analogs of $L^{2}$-Betti numbers there is an approximation result similar to Theorem 1.1:

Theorem 1.4. Let $d=\operatorname{dim}(G)$. Then for any integer $k$ and as $i$ tends to infinity, we have

$$
b_{k}\left(X_{i} ; \mathbb{F}_{p}\right)=\beta_{k}\left(\bar{X}, \bar{\Gamma} ; \mathbb{F}_{p}\right)\left[\Gamma: \Gamma_{i}\right]+O\left(\left[\Gamma: \Gamma_{i}\right]^{1-1 / d}\right) .
$$

In particular, the limit of the sequence $b_{k}\left(X_{i} ; \mathbb{F}_{p}\right) /\left[\Gamma: \Gamma_{i}\right]$ exists and is equal to $\beta_{k}\left(\bar{X}, \bar{\Gamma} ; \mathbb{F}_{p}\right)$.

Here again Calegari informed us that Theorem 1.4 can be deduced from his joint ongoing work with Emerton on completed cohomology. In fact one key feature of their theory is to set up the right framework to determine the growth rate of $(\bmod p)$ Betti numbers even if the corresponding $(\bmod p) L^{2}$-Betti number vanishes. Proving unconditional results seems difficult; we nevertheless point out that when $X$ is 3dimensional the main result of [6] implies in particular that the error term in Theorem 1.4 is the best possible in general. We also note that - in his PhD-thesis [30], Theorem 5.3.1 - Liam Wall had first constructed examples of $p$-adic analytic towers of covers of a finite volume hyperbolic 3-manifold which shows that one cannot replace the error term $O\left(\left[\Gamma: \Gamma_{i}\right]^{1-1 / d}\right)$ by $O\left(\left[\Gamma: \Gamma_{i}\right]^{1-1 / d-\epsilon}\right)$ for some $\epsilon>0$.

We may have $\beta_{k}(\bar{X}, \bar{\Gamma}) \neq \beta_{k}\left(\bar{X}, \bar{\Gamma} ; \mathbb{F}_{p}\right)$. An example is given in [23] (Example 6.2). One can even construct an example with $X$ being a manifold (see Section 5): 
Proposition 1.5. There exists a link complement $X$ and a sequence of $p$-covers $X_{i}$ of $X$ such that

$$
\lim _{i \rightarrow+\infty} \frac{\operatorname{dim} H_{1}\left(X_{i}, \mathbb{F}_{p}\right)}{\left[\Gamma: \Gamma_{i}\right]} \neq \lim _{i \rightarrow+\infty} \frac{\operatorname{dim} H_{1}\left(X_{i}, \mathbb{Q}\right)}{\left[\Gamma: \Gamma_{i}\right]}
$$

We don't know of any example with $\bar{X}$ being aspherical.

1.4. Beyond $p$-adic analytic groups. The following theorem about arbitrary pro-p towers is certainly known to some experts but we could find no proof in the literature except in degree one.

Theorem 1.6. Let $k$ be a field of characteristic $p>0$. Let $X$ be a compact connected $C W$-complex with $\Gamma$ as fundamental group. Let $\left(\Gamma_{i}\right)_{i \geq 0}$ be a residual p-chain. We denote the finite cover of $X$ associated to $\Gamma_{i}$ by $X_{i}$. Then, for any $n \geq 0$, the sequence of normalized Betti numbers with $k$-coefficients

$$
\left(\frac{b_{n}\left(X_{i} ; k\right)}{\left[\Gamma: \Gamma_{i}\right]}\right)_{i \geq 0}
$$

is monotone decreasing and converges as $i \rightarrow \infty$.

We moreover prove that the limit is an integer in many situations, see Theorem 4.3 and the remark following it.

1.5. Acknowledgments. We thank the referee for a detailed and helpful report, especially for spotting an error in a previous version of the proof of Theorem 2.1, which is now corrected.

Work on this project was supported by the Leibniz Award of W.L. granted by the DFG. N.B. is a member of the Institut Universitaire de France. P.L. was partially supported by a grant from the NSA. R.S. thanks the Mittag-Leffler institute for its hospitality during the final stage of this project and acknowledges support by grant SA $1661 / 3-1$ of the DFG.

\section{Proof of Theorem 1.1 and 1.4}

In the sequel we treat the cases $R=\mathbb{Z}_{p}$ and $R=\mathbb{F}_{p}$ simultaneously. Depending on which case, we denote by $\operatorname{dim}_{R}(M)$ either the dimension of a $\mathbb{F}_{p}$-vector space or the $\mathbb{Z}_{p}$-rank of a $\mathbb{Z}_{p}$-module, which equals the dimension of the $\mathbb{Q}_{p}$-vector space $\mathbb{Q}_{p} \otimes_{\mathbb{Z}_{p}} M$.

As in the work of Calegari-Emerton and Emerton [5], [6] the following result of M. Harris ([18], Theorem 1.10) for which corrections appear in [19], is crucial. 
Although not explicitly stated as such, a proof is also contained in the work of FarkasLinnell [16]. We give a complete proof blending ideas from both Farkas-Linnell's and Harris' papers.

Theorem 2.1 (Harris). Let $R=\mathbb{Z}_{p}$ or $R=\mathbb{F}_{p}$. Let $M$ be a finitely generated $R[[G]]$-module. Then

$$
\operatorname{dim}_{R}\left(R \hat{\otimes}_{R\left[\left[G_{i}\right]\right]} M\right)=\operatorname{rank}_{R[[G]]}(M)\left[G: G_{i}\right]+O\left(\left[G: G_{i}\right]^{1-1 / d}\right) .
$$

Here $\hat{\otimes}$ denotes the completed tensor product.

Proof. Passing to a finite index subgroup of $G$ we may, and shall, assume that $G$ is uniform and torsion-free. The proof then proceeds through a sequence of reductions.

Reduction to the case of cokernels of elements in $R[[G]]$. We first show that it suffices to show the theorem for $R[[G]]$-modules of the form

$$
M=\operatorname{coker}(R[[G] \stackrel{\stackrel{-a}{\longrightarrow}}{\longrightarrow} R[[G]]),
$$

with $a \in R[[G]]$. Let $N$ be an arbitrary finitely generated $R[[G]]$-module. Since $R[[G]]$ is Noetherian, $N$ is finitely presented and we can find a matrix $A \in M(r \times$ $s, R[[G]])$ such that

$$
N=\operatorname{coker}\left(R[[G]]^{r} \stackrel{r_{A}}{\longrightarrow} R[[G]]^{s}\right),
$$

where $r_{A}$ denotes the right multiplication

$$
r_{A}\left(x_{1}, \ldots, x_{r}\right)=\left(x_{1}, \ldots, x_{r}\right) \cdot A
$$

with $A$. Since the Ore localization $Q(R[[G]])$ is a skew field, by row and column reduction in $M_{r}(Q(R[[G]]))$ one can find invertible matrices $B \in M(r \times r, Q(R[[G]]))$ and $C \in M(s \times s, Q(R[[G]]))$ such that $D:=B A C$ is a block matrix of the form

$$
D=\left(\begin{array}{cc}
I_{\delta} & 0_{\delta, s-\delta} \\
0_{r-\delta, \delta} & 0_{r-\delta \times s-\delta}
\end{array}\right) \in M(r \times s, R[[G]]),
$$

where $I_{\delta} \in M(\delta \times \delta, R[[G]])$ is the identity matrix with $\delta \in\{0, \ldots, s\}$ and the other blocks are suitable zero matrices. In other words, $D$ describes the projection onto the first $\delta$ coordinates $\left(x_{1}, x_{2}, \ldots, x_{r}\right) \mapsto\left(x_{1}, x_{2}, \ldots, x_{\delta}, 0,0, \ldots\right)$. Since $B, C$ are invertible, we have

$$
\operatorname{rank}_{R[[G]]}(N)=s-\delta .
$$

There are nonzero $b, c \in R[[G]]$ such that $b B, C c$ are matrices over $R[[G]]$. We have

$$
\text { (bB) } A(C c)=\left(\begin{array}{cc}
b c \cdot I_{\delta} & 0_{\delta, s-\delta} \\
0_{r-\delta, \delta} & 0_{\delta \times \delta}
\end{array}\right) .
$$


Let $A_{i}, B_{i}$, and $C_{i}$ be the mod $G_{i}$ reductions of $A, b B$, and $C c$. Because of $\operatorname{ker}\left(r_{A_{i}}\right) \subset$ $\operatorname{ker}\left(r_{C_{i}} \circ r_{A_{i}}\right)=\operatorname{ker}\left(r_{A_{i} C_{i}}\right)$ one obtains $\operatorname{dim}_{R} \operatorname{ker}\left(r_{A_{i}}\right) \leq \operatorname{dim}_{R} \operatorname{ker}\left(r_{A_{i} C_{i}}\right)$. Because of $\operatorname{im}\left(r_{B_{i} A_{i} C_{i}}\right)=\operatorname{im}\left(r_{A_{i} C_{i}} \circ r_{B_{i}}\right) \subset \operatorname{im}\left(r_{A_{i} C_{i}}\right)$ we have $\operatorname{dim}_{R} \operatorname{ker}\left(r_{A_{i} C_{i}}\right) \leq$ $\operatorname{dim}_{R} \operatorname{ker}\left(r_{B_{i} A_{i} C_{i}}\right)$. Therefore: $\operatorname{dim}_{R} \operatorname{ker}\left(r_{A_{i}}\right) \leq \operatorname{dim}_{R} \operatorname{ker}\left(r_{B_{i} A_{i} C_{i}}\right)$. Assuming the theorem is proved for modules as in (2.2), this implies that

$$
\begin{aligned}
\operatorname{dim}_{R}\left(R \hat{\otimes}_{R\left[\left[G_{i}\right]\right]} N\right) & =\operatorname{dim}_{R} \operatorname{coker}\left(r_{A_{i}}\right) \\
& =\operatorname{dim}_{R} \operatorname{ker}\left(r_{A_{i}}\right)-(r-s)\left[G: G_{i}\right] \\
& \leq \operatorname{dim}_{R} \operatorname{ker}\left(r_{B_{i} A_{i} C_{i}}\right)-(r-s)\left[G: G_{i}\right] \\
& =\operatorname{dim}_{R} \operatorname{coker}\left(r_{B_{i} A_{i} C_{i}}\right) \\
& =(s-\delta) \cdot\left[G: G_{i}\right]+O\left(\left[G: G_{i}\right]^{1-1 / d}\right) .
\end{aligned}
$$

To prove the assertion for $N$, under the assumption that it holds for modules as in (2.2), it remains to show that

$$
\operatorname{dim}_{R} \operatorname{coker}\left(r_{A_{i}}\right) \geq(s-\delta)\left[G: G_{i}\right]-O\left(\left[G: G_{i}\right]^{1-1 / d}\right) .
$$

Let $E \in M((r-\delta) \times r, R[[G]])$ be the matrix such that

$$
R[[G]]^{r-\delta} \stackrel{r_{E}}{\longrightarrow} R[[G]]^{r}, \quad\left(y_{1}, y_{2}, \ldots, y_{r-\delta}\right) \cdot E=\left(0, \ldots, 0, y_{1}, y_{2}, \ldots, y_{r-\delta}\right) .
$$

Let $F \in M((r-\delta) \times r, R[[G]])$ be the matrix $E \cdot(b B)$. The same argument as before leading to (2.5) but now applied to $F$ shows

$$
\operatorname{dim}_{R} \operatorname{coker}\left(r_{F_{i}}\right) \leq \delta \cdot\left[G: G_{i}\right]+O\left(\left[G: G_{i}\right]^{1-1 / d}\right) .
$$

We have $0=r_{D} \circ r_{E}=r_{E D}$, yielding $E D=0$ and

$$
F A C=E(b B) A C=E\left(b I_{r}\right) B A C=\left(b I_{r}\right) E B A C=\left(b I_{r-\delta}\right) E D=0 .
$$

Note that we used $E\left(b I_{r}\right)=\left(b I_{r-\delta}\right) E$ here. As $C$ is invertible this implies $F A=0$ and $r_{A} \circ r_{F}=r_{F A}=0$. In particular, $r_{A_{i}} \circ r_{F_{i}}=0$ and hence $\operatorname{im}\left(r_{F_{i}}\right) \subseteq \operatorname{ker}\left(r_{A_{i}}\right)$. So $\operatorname{dim}_{R} \operatorname{im}\left(r_{F_{i}}\right) \leq \operatorname{dim}_{R} \operatorname{ker}\left(r_{A_{i}}\right)$. We compute

$$
\begin{aligned}
& \operatorname{dim}_{R} \operatorname{coker}\left(r_{A_{i}}\right)+\operatorname{dim}_{R} \operatorname{coker}\left(r_{F_{i}}\right) \\
& \quad=s \cdot\left[G: G_{i}\right]-\operatorname{dim}_{R} \operatorname{im}\left(r_{A_{i}}\right)+r \cdot\left[G: G_{i}\right]-\operatorname{dim}_{R} \operatorname{im}\left(r_{F_{i}}\right) \\
& \quad \geq s \cdot\left[G: G_{i}\right]-\operatorname{dim}_{R} \operatorname{im}\left(r_{A_{i}}\right)+r \cdot\left[G: G_{i}\right]-\operatorname{dim}_{R} \operatorname{ker}\left(r_{A_{i}}\right) \\
& \quad=s \cdot\left[G: G_{i}\right] .
\end{aligned}
$$

Now (2.6) follows from (2.7).

Reduction to the case $R=\mathbb{F}_{p}$. To prove the statement for a finitely generated $\mathbb{Z}_{p}[[G]]$-module $M$ we may assume that $\operatorname{rank}_{\mathbb{Z}_{p}[[G]]}(M)=0$ due to the reduction to the case (2.2) and the fact that $\mathbb{Z}_{p}[[G]]$ has no zero-divisors. For the following reason we may, in addition, assume that $M$ has no $p$-torsion: Let $T=\{m \in M \mid$ 
$\left.\exists_{d(m) \in \mathbb{N}} p^{d(m)} \cdot m=0\right\}$ be its $p$-torsion part. Obviously, $T$ is a $\mathbb{Z}_{p}[[G]]$-submodule of $M$. One easily sees by additivity of dimension that

and

$$
\operatorname{dim}_{\mathbb{Q}_{p}}\left(\mathbb{Q}_{p} \hat{\otimes}_{\mathbb{Z}_{p}\left[\left[G_{i}\right]\right]} M / T\right)=\operatorname{dim}_{\mathbb{Q}_{p}}\left(\mathbb{Q}_{p} \hat{\otimes}_{\mathbb{Z}_{p}\left[\left[G_{i}\right]\right]} M\right)
$$

$$
\operatorname{rank}_{\mathbb{Z}_{p}[[G]]}(M)=\operatorname{rank}_{\mathbb{Z}_{p}[[G]]}(M / T) .
$$

Hence we may assume that $M$ has no $p$-torsion. We prove now that

$$
\operatorname{rank}_{\mathbb{F}_{p}[[G]]}(M / p M)=\operatorname{rank}_{\mathbb{Z}_{p}[[G]]}(M),
$$

hence both are zero. Since the ring $\mathbb{Z}_{p}[[G]]$ has finite projective dimension ([3], Section 5.1), and every projective $\mathbb{Z}_{p}[[G]]$-module is free, see [32], Corollary 7.5.4 on p. 127 , and $\mathbb{Z}_{p}[[G]]$ is Noetherian, the finitely generated $\mathbb{Z}_{p}[[G]]$-module $M$ possesses a finite resolution by finitely generated free $\mathbb{Z}_{p}[[G]]$-modules:

$$
0 \rightarrow F_{n} \rightarrow F_{n-1} \rightarrow \cdots \rightarrow F_{0} \rightarrow M \rightarrow 0 .
$$

Applying the functor $N \mapsto \mathbb{F}_{p} \otimes_{\mathbb{Z}} N \cong N / p N$ yields a resolution of $M / p M$ by finitely generated, free $\mathbb{F}_{p}[[G]]$-modules since $M$ has no $p$-torsion:

$$
0 \rightarrow \mathbb{F}_{p} \otimes_{\mathbb{Z}} F_{n} \rightarrow \cdots \rightarrow \mathbb{F}_{p} \otimes_{\mathbb{Z}} F_{0} \rightarrow M / p M \rightarrow 0 .
$$

Now equation (2.8) follows since the rank functions over $\mathbb{F}_{p}[[G]]$ and $\mathbb{Z}_{p}[[G]]$ are additive and the equation obviously holds for finitely generated free $\mathbb{Z}_{p}[[G]]$-modules.

Let $N=\mathbb{Z}_{p} \hat{\otimes}_{\mathbb{Z}_{p}\left[\left[G_{i}\right]\right]} M$. Because of $\operatorname{rank}_{\mathbb{Z}_{p}[[G]]}(M)=0$ it is enough to prove $\operatorname{dim}_{\mathbb{Z}_{p}}(N)=O\left(\left[G: G_{i}\right]^{1-1 / d}\right)$. This follows from the $\mathbb{F}_{p}[[G]]$-case, $\operatorname{rank}_{\mathbb{F}_{p}[[G]]}(M / p M)=0$, and the inequality

$$
\operatorname{dim}_{\mathbb{Z}_{p}}(N) \leq \operatorname{dim}_{\mathbb{F}_{p}}(N / p N)=\operatorname{dim}_{\mathbb{F}_{p}}\left(\mathbb{F}_{p} \hat{\otimes}_{\mathbb{F}_{p}\left[\left[G_{i}\right]\right]} M / p M\right) .
$$

So we reduced the proof of the theorem to the case $R=\mathbb{F}_{p}$ and henceforth assume $R=\mathbb{F}_{p}$.

Reduction to $G$ being standard. We finally reduce the assertion to the case that $G$ is standard in the sense of [12], §8.4. Being a $p$-adic analytic group, $G$ has an open subgroup $H$ which is standard with respect to the manifold structure induced from $G$, see Theorem 8.29 of [12]. Since $H$ is open, we have $G_{i}<H$ for $i$ greater than some $i_{0}$. Being standard $H$ has a preferred collection of open normal subgroups $H_{i}$ which satisfy: $G_{i_{0}+i-1} \subset H_{i} \subset G_{i}(i \geq 1)$; see e.g., [12], Example 6, p. 168.

Recall that we may assume that $\operatorname{rank}_{\mathbb{F}_{p}[[G]]}(M)=0$ due to the reduction to the case (2.2) and the fact that $\mathbb{F}_{p}[[G]]$ has no zero-divisors.

Now if the assertion holds for $H$ with respect to the $H_{i}$ 's, then it follows that for $i>$ $i_{0}$ the left-hand side of (2.1) is bounded by a constant times $\operatorname{dim}_{R}\left(R \hat{\otimes}_{R\left[\left[H_{i-i_{0}+1}\right]\right]} M\right)$ and is therefore $O\left(\left[H: H_{i}\right]^{1-1 / d}\right)=O\left(\left[G: G_{i}\right]^{1-1 / d}\right)$, so the assertion holds for 
$G$ as well. We assume from now on that $G$ is standard and that $G_{i}=\psi^{-1}\left(p^{i} \mathbb{Z}_{p}^{d}\right)$ where $\psi$ is the global atlas of $G$.

The remaining argument. Let $M$ be as in (2.2). We may assume $a \neq 0$. By a fundamental result of Lazard, the graded ring $\mathrm{gr} \mathrm{F}_{p}[[G]]$ with respect to the filtration $\left(\Delta^{n}\right)_{n \geq 0}$ by powers of the augmentation ideal $\Delta \subset \mathbb{F}_{p}[[G]]$ is a polynomial algebra $\mathbb{F}_{p}\left[X_{1}, \ldots, X_{d}\right]$ with indeterminates $X_{i}=x_{i}-1+\Delta^{2}$ ([32], Theorem 8.7.10 on p. 160) where $\left\{x_{1}, \ldots, x_{d}\right\} \subset G$ is a minimal generating set. Let $I_{i} \subset \mathbb{F}_{p}[[G]]$ be the closure of the ideal generated by elements $\lambda(h-1)$ with $h \in G_{i}$. Note that $N / I_{i} N \cong \mathbb{F}_{p} \hat{\otimes}_{\mathbb{F}_{p}\left[\left[G_{i}\right]\right]} N$ for any $\mathbb{F}_{p}[[G]]$-module $N$. Since $\mathbb{F}_{p}[[G]]$ is a domain, $\operatorname{rank}_{\mathbb{F}_{p}[[G]]}(M)=0$. Now for each integer $i \geq 1$ (if $p>2$ ) or $i \geq 2$ (if $p=2$ ), the global atlas $\psi$ of $G$ induces an epimorphism $G_{i} \rightarrow p^{i} \mathbb{Z}_{p}^{d} / p^{i+1} \mathbb{Z}_{p}^{d}$ with kernel $G_{i+1}$. It therefore follows that $\left[G: G_{i}\right]=C p^{i d}$ for some rational constant $C>0$ and we have to show that

$$
\operatorname{dim}_{\mathbb{F}_{p}}\left(M / I_{i} M\right)=O\left(p^{(d-1) i}\right) .
$$

But it follows from Lemma 7.1 of [12] that there exists a positive integer $m$ such that $\Delta^{m p^{i}} \subset I_{i}$ for all $i$. It therefore suffices to show (2.9) with $I_{i}$ replaced by $\Delta^{m p^{i}}$. Let $s \geq 0$ be such that $a \in \Delta^{s} \backslash \Delta^{s+1}$. Let $a_{i}: \mathbb{F}_{p}[[G]] / \Delta^{m p^{i}} \rightarrow \mathbb{F}_{p}[[G]] / \Delta^{m p^{i}}$ be the map induced by right multiplication with $a$. We have

$$
\begin{aligned}
\operatorname{dim}_{\mathbb{F}_{p}}\left(M / \Delta^{m p^{i}} M\right) & =\operatorname{dim}_{\mathbb{F}_{p}} \operatorname{coker}\left(a_{i}\right) \\
& =\operatorname{dim}_{\mathbb{F}_{p}} \operatorname{ker}\left(a_{i}\right) \\
& =\operatorname{dim}_{\mathbb{F}_{p}}\left(\Delta^{m p^{i}-s} / \Delta^{m p^{i}}\right) .
\end{aligned}
$$

The last equality follows from the fact the graded ring is a polynomial ring. For the same reason the last number equals the number of monomials in a polynomial ring with $d$ variables each of which has total degree in the interval $\left[m p^{i}-s, m p^{i}\right)$. The number of monomials of degree $<k$ is $\left(\begin{array}{c}d+k-1 \\ d\end{array}\right)$. Hence

$$
\operatorname{dim}_{\mathbb{F}_{p}}\left(M / \Delta^{m p^{i}} M\right)=\left(\begin{array}{c}
d+m p^{i}-1 \\
d
\end{array}\right)-\left(\begin{array}{c}
d+m p^{i}-s-1 \\
d
\end{array}\right) .
$$

As a polynomial in $p^{i}$, each binomial coefficient has leading term $\left(m p^{i}\right)^{d}$. Their difference is a polynomial in $p^{i}$ with degree at most $d-1$. This implies (2.9).

Proofs of Theorems 1.1 and 1.4. We show for both cases $R=\mathbb{Z}_{p}$ and $R=\mathbb{F}_{p}$ simultaneously that

$$
b_{k}\left(X_{i} ; R\right)=\operatorname{rank}_{R[[G]]}\left(H_{k}\left(R[[G]] \otimes_{R \bar{\Gamma}} C_{*}(\bar{X})\right)\right) \cdot\left[\Gamma: \Gamma_{i}\right]+O\left(\left[\Gamma: \Gamma_{i}\right]^{1-1 / d}\right) .
$$

The CW-structure on $X$ lifts to a $\bar{\Gamma}$-equivariant CW-structure on $\bar{X}$ and to $\Gamma_{i} \backslash \Gamma$ equivariant CW-structures on $X_{i}$. We may also view $\bar{X}$ as a $\Gamma$-space via the quotient 
map $\Gamma \rightarrow \bar{\Gamma}$. Let $C_{*}(\bar{X})$ be the cellular chain complex of $\bar{X}$. Each chain module $C_{k}(\bar{X})$ is a finitely generated free $\mathbb{Z}[\bar{\Gamma}]$-module. The differentials in the chain complex $C_{*}=R \otimes_{\mathbb{Z}} C_{*}(\bar{X})$ are denoted by $\partial_{*}$. Note that $R \otimes_{R\left[\Gamma_{i}\right]} C_{*}$ is isomorphic to the cellular chain complex $R \otimes_{\mathbb{Z}} C_{*}\left(X_{i}\right)$ as an $R\left[\Gamma_{i} \backslash \Gamma\right]$-chain complex. In particular, we have

$$
H_{*}\left(X_{i}, R\right) \cong H_{*}\left(R \otimes_{R\left[\Gamma_{i}\right]} C_{*}\right) .
$$

We write $\hat{C}_{*}$ and $\hat{\partial}_{*}$ short for $R[[G]] \otimes_{R \bar{\Gamma}} C_{*}$ and its differentials. We denote the cycles and boundaries in the chain complexes $\hat{C}_{*}$ and $C_{*}$ by $\hat{Z}_{*}, \hat{B}_{*}$ and $Z_{*}, B_{*}$, respectively. Let $r_{n} \in \mathbb{N}_{0}$ be the rank of the finitely generated free $R[[G]]$-module $\widehat{C}_{n}$. In each degree $n$ we have the obvious exact sequence

$$
0 \rightarrow \hat{Z}_{n} \rightarrow \hat{C}_{n} \stackrel{\hat{\partial}_{n}}{\longrightarrow} \widehat{C}_{n-1} \rightarrow \operatorname{coker}\left(\hat{\partial}_{n}\right) \rightarrow 0 .
$$

By additivity of $\operatorname{rank}_{R[[G]]}$ we obtain that

$$
\begin{aligned}
\operatorname{rank}_{R[[G]]}\left(H_{k}\left(\hat{C}_{*}\right)\right)= & \operatorname{rank}_{R[G]]}\left(\hat{Z}_{k} / \hat{B}_{k}\right) \\
= & \operatorname{rank}_{R[[G]]}\left(\hat{Z}_{k}\right)-\operatorname{rank}_{R[[G]]}\left(\hat{B}_{k}\right) \\
= & r_{k}-r_{k-1}+\operatorname{rank}_{R[[G]]}\left(\operatorname{coker}\left(\hat{\partial}_{k}\right)\right)-\operatorname{rank}_{R[[G]]}\left(\hat{B}_{k}\right) \\
= & r_{k}-r_{k-1}+\operatorname{rank}_{R[[G]]}\left(\operatorname{coker}\left(\hat{\partial}_{k}\right)\right) \\
& \quad-\left(r_{k}-\operatorname{rank}_{R[[G]]}\left(\operatorname{coker}\left(\hat{\partial}_{k+1}\right)\right)\right) \\
= & \operatorname{rank}_{R[[G]]}\left(\operatorname{coker}\left(\hat{\partial}_{k}\right)\right)+\operatorname{rank}{ }_{R[[G]]}\left(\operatorname{coker}\left(\hat{\partial}_{k+1}\right)\right)-r_{k-1} .
\end{aligned}
$$

By (2.11), a similar argument as above, and right-exactness of the tensor product, we obtain that

$$
\begin{aligned}
\operatorname{dim}_{R}\left(H_{k}\left(X_{i}, R\right)\right)= & \operatorname{dim}_{R}\left(H_{k}\left(R \otimes_{R\left[\Gamma_{i}\right]} C_{*}\right)\right) \\
= & \operatorname{dim}_{R}\left(\operatorname{coker}\left(R \otimes_{R\left[\Gamma_{i}\right]} \partial_{k}\right)\right) \\
& \quad+\operatorname{dim}_{R}\left(\operatorname{coker}\left(R \otimes_{R\left[\Gamma_{i}\right]} \partial_{k+1}\right)\right)-\left[\Gamma: \Gamma_{i}\right] \cdot r_{k-1} \\
= & \operatorname{dim}_{R}\left(R \otimes_{R\left[\Gamma_{i}\right]} \operatorname{coker}\left(\partial_{k}\right)\right) \\
& \quad+\operatorname{dim}_{R}\left(R \otimes_{R\left[\Gamma_{i}\right]} \operatorname{coker}\left(\partial_{k+1}\right)\right)-\left[\Gamma: \Gamma_{i}\right] \cdot r_{k-1} .
\end{aligned}
$$

Hence,

$$
\frac{b_{k}\left(X_{i}, R\right)}{\left[\Gamma: \Gamma_{i}\right]}=\frac{\operatorname{dim}_{R}\left(R \otimes_{R\left[\Gamma_{i}\right]} \operatorname{coker}\left(\partial_{k}\right)\right)}{\left[\Gamma: \Gamma_{i}\right]}+\frac{\operatorname{dim}_{R}\left(R \otimes_{R\left[\Gamma_{i}\right]} \operatorname{coker}\left(\partial_{k+1}\right)\right)}{\left[\Gamma: \Gamma_{i}\right]}-r_{k-1} .
$$

The natural map

$$
R \otimes_{R\left[\Gamma_{i}\right]} R[\Gamma] \stackrel{\cong}{\longrightarrow} R \hat{\otimes}_{R\left[\left[G_{i}\right]\right]} R[[G]]
$$

induced by $\phi: \Gamma \rightarrow G$ is a right $R[\Gamma]$-module isomorphism (recall that we regard $R[[G]]$ as a right $R[\Gamma]$-module via $\phi)$. The inverse is obtained as follows: Since 
$\Gamma_{i} \backslash \Gamma \cong G_{i} \backslash G$, there is a natural continuous homomorphism from $G$ to the invertible elements of the $R$-algebra $R \otimes_{R\left[\Gamma_{i}\right]} R[\Gamma]$. By the universal property of the completed group algebra there is a continuous homomorphism $R[[G]] \rightarrow R \otimes_{R\left[\Gamma_{i}\right]} R[\Gamma]$ which descends to the desired inverse. As a consequence we get isomorphisms

$$
\begin{aligned}
R \hat{\otimes}_{R\left[\left[G_{i}\right]\right]} \operatorname{coker}\left(\hat{\partial}_{k}\right) & \cong R \otimes_{R\left[\left[G_{i}\right]\right]} R[[G]] \otimes_{R[\bar{\Gamma}]} \operatorname{coker}\left(\partial_{k}\right) \\
& \cong R \otimes_{R\left[\left[G_{i}\right]\right]} R[[G]] \otimes_{R[\Gamma]} \operatorname{coker}\left(\partial_{k}\right) \\
& \cong R \otimes_{R\left[\Gamma_{i}\right]} \operatorname{coker}\left(\partial_{k}\right)
\end{aligned}
$$

and, thus,

$$
\begin{aligned}
\frac{b_{k}\left(X_{i}, R\right)}{\left[G: G_{i}\right]}= & \frac{\operatorname{dim}_{R}\left(R \hat{\otimes}_{R\left[\left[G_{i}\right]\right]} \operatorname{coker}\left(\hat{\partial}_{k}\right)\right)}{\left[G: G_{i}\right]} \\
& +\frac{\operatorname{dim}_{R}\left(R \hat{\otimes}_{R\left[\left[G_{i}\right]\right]} \operatorname{coker}\left(\hat{\partial}_{k+1}\right)\right)}{\left[G: G_{i}\right]}-r_{k-1} .
\end{aligned}
$$

Now (2.10) follows from (2.12), (2.14), and Theorem 2.1. Note that (2.10) is exactly the statement of Theorem 1.4 in the case $R=\mathbb{F}_{p}$. Next we explain how Theorem 1.1 follows from (2.10) when $R=\mathbb{Z}_{p}$. Since $\mathbb{Q}_{p}$ has characteristic zero, we have $b_{k}\left(X_{i}\right)=b_{k}\left(X_{i}, \mathbb{Q}_{p}\right)=b_{k}\left(X_{i}, \mathbb{Z}_{p}\right)$. Since $b_{k}\left(X_{i}\right) /\left[\Gamma: \Gamma_{i}\right] \rightarrow \beta_{k}(\bar{X}, \bar{\Gamma})$ as $i \rightarrow \infty$ [24], we conclude

$$
\beta_{k}(\bar{X}, \bar{\Gamma})=\operatorname{rank}_{\mathbb{Z}_{p}[[G]]}\left(H_{k}\left(\mathbb{Z}_{p}[[G]] \otimes_{\mathbb{Z}_{p} \bar{\Gamma}} C_{*}\right)\right) .
$$

\section{Relation with the completed homology}

Calegari and Emerton [7], [6] have introduced the completed homology groups:

$$
\tilde{H}_{k}=\lim _{\leftarrow} H_{k}\left(X_{i}, \mathbb{Z}_{p}\right) \quad \text { and } \quad \tilde{H}_{k}\left(\mathbb{F}_{p}\right)=\lim _{\longleftarrow} H_{k}\left(X_{i}, \mathbb{F}_{p}\right) .
$$

These modules carry continuous actions of $G$ and may therefore be considered as $\mathbb{Z}_{p}[[G]]$-modules or $\mathbb{F}_{p}[[G]]$-modules, respectively. In this section we want to clarify the relation of completed cohomology to $(\bmod p) L^{2}$-Betti numbers.

Proposition 3.1. Retaining the setup in Section 1.1 we have

$$
\beta_{k}\left(\bar{X}, \bar{\Gamma} ; \mathbb{F}_{p}\right)=\operatorname{rank}_{\mathbb{F}_{p}[[G]]}\left(\widetilde{H}_{k}\left(\mathbb{F}_{p}\right)\right) .
$$

Proof. Here again we may reduce to the case where $G$ is torsion-free. Write $C_{*}=$ $C_{*}\left(\bar{X} ; \mathbb{F}_{p}\right)$. The claim is equivalent to

$$
\tilde{H}_{k}\left(\mathbb{F}_{p}\right)=\lim _{\longleftarrow} H_{k}\left(\mathbb{F}_{p} \otimes_{\mathbb{F}_{p}\left[\Gamma_{i}\right]} C_{*}\right)
$$


and

$$
H_{k}\left(\mathbb{F}_{p}[[G]] \otimes_{\mathbb{F}_{p} \bar{\Gamma}} C_{*}\right)=H_{k}\left(\lim ^{\longleftarrow}\left(\mathbb{F}_{p} \otimes_{\mathbb{F}_{p}\left[\Gamma_{i}\right]} C_{*}\right)\right)
$$

having the same $\mathbb{F}_{p}[[G]]$-rank. So the statement is equivalent to:

$$
\begin{aligned}
Q\left(\mathbb{F}_{p}[[G]]\right) \otimes_{\mathbb{F}_{p}[[\Gamma]]} H_{k}\left(\lim _{\leftarrow}\left(\mathbb{F}_{p} \otimes_{\mathbb{F}_{p}\left[\Gamma_{i}\right]} C_{*}\right)\right) \\
\cong Q\left(\mathbb{F}_{p}[[G]]\right) \otimes_{\mathbb{F}_{p}[[\Gamma]]}\left(\lim _{\leftarrow} H_{k}\left(\mathbb{F}_{p} \otimes_{\mathbb{F}_{p}\left[\Gamma_{i}\right]} C_{*}\right)\right) .
\end{aligned}
$$

Since $\mathbb{F}_{p} \otimes_{\mathbb{Z}_{p}\left[\Gamma_{i}\right]} C_{*}$ is a tower of chain complexes of abelian groups satisfying the Mittag-Leffler condition, by Theorem 3.5.8 of [31] there is a short exact sequence

$$
\begin{aligned}
0 \rightarrow \lim ^{1} H_{k+1}\left(\mathbb{F}_{p} \otimes_{\mathbb{F}_{p}\left[\Gamma_{i}\right]} C_{*}\right) \rightarrow H_{k}\left(\lim _{\leftarrow}\left(\mathbb{F}_{p} \otimes_{\mathbb{F}_{p}\left[\Gamma_{i}\right]} C_{*}\right)\right) \\
\rightarrow \underset{\leftarrow}{\lim } H_{k}\left(\mathbb{F}_{p} \otimes_{\mathbb{F}_{p}\left[\Gamma_{i}\right]} C_{*}\right) \rightarrow 0 .
\end{aligned}
$$

Moreover, since towers of finite dimensional vector spaces over a field satisfy the Mittag-Leffler condition, we conclude that

$$
\lim ^{1} H_{k+1}\left(\mathbb{F}_{p} \otimes_{\mathbb{F}_{p}\left[\Gamma_{i}\right]} C_{*}\right)=0,
$$

which yields the proposition.

It follows from works of Calegari and Emerton that a similar result with $\mathbb{F}_{p}$ replaced by $\mathbb{Z}_{p}$ holds as well, that is,

$$
\beta_{k}\left(\bar{X}, \bar{\Gamma} ; \mathbb{Z}_{p}\right)=\operatorname{rank}_{\mathbb{Z}_{p}[[G]]}\left(\tilde{H}_{k}\right),
$$

and we want to indicate why. In [5], Theorem 3.2, it is shown for arithmetic congruence covers $X_{i}$ of symmetric spaces that the so-called co-rank $r_{k}$ of the completed cohomology $\widetilde{H}^{k}$ satisfies the equality in Theorem 1.1 with $\beta_{k}(\bar{X}, \bar{\Gamma})$ replaced by $r_{k}$. The proof in [5] is a consequence of a general result of Emerton [14], Theorem 2.1.5, and their Lemma 2.2. Both these results hold not only for arithmetic congruence covers but in our generality. Since the co-rank of $\widetilde{H}^{k}$ is the same as the rank of $\widetilde{H}_{k}$, see [7], Theorem 1.1 (3), this implies (3.1).

It is somewhat harder to work with completed homology, see [5], [6]. We nevertheless want to emphasize that the latter contains a lot more information. It should be the right framework to determine the growth rate of $(\bmod p)$ Betti numbers even if the corresponding $(\bmod p) L^{2}$-Betti number vanishes. However it seems hard to extract the necessary information from completed homology, the only exception that we are aware of is in case $X$ is 3-dimensional, see [6].

\section{Approximation results for pro- $p$ towers that are not $p$-adic analytic}

The proof of Theorem 1.6 relies on the following well-known lemma (see also [15] for a proof). 
Lemma 4.1. Let $k$ be a field of characteristic $p>0$. Let $\Lambda$ be a normal subgroup in a group $\Gamma$ whose index is a p-power. Then

$$
\operatorname{dim}_{k}\left(k \otimes_{k[\Lambda]} M\right) \leq[\Gamma: \Lambda] \cdot \operatorname{dim}_{k}\left(k \otimes_{k[\Gamma]} M\right) .
$$

Proof. Because of the isomorphism

$$
k \otimes_{k[\Gamma]} M \cong k \otimes_{k[\Gamma / \Lambda]}\left(k \otimes_{k[\Lambda]} M\right)
$$

it suffices to prove the case where $\Lambda$ is trivial and $\Gamma$ is a finite $p$-group. Let

$$
k[\Gamma]^{m} \stackrel{f}{\rightarrow} k[\Gamma]^{n} \rightarrow M \rightarrow 0
$$

be a presentation of $M$. Then $\bar{f}=k \otimes_{k[\Gamma]} f$ is a presentation of $k \otimes_{k[\Gamma]} M$. Since $\operatorname{dim}_{k}(M)=|\Gamma| \cdot n-\operatorname{dim}_{k}(\operatorname{im}(f))$ and $\operatorname{dim}_{k}\left(k \otimes_{k[\Gamma]} M\right)=n-\operatorname{dim}_{k}(\operatorname{im}(\bar{f}))$, we have to show that

$$
\operatorname{dim}_{k}(\operatorname{im}(f)) \geq|\Gamma| \cdot \operatorname{dim}_{k}(\operatorname{im}(\bar{f})) .
$$

Extend a $k$-basis $\left\{u_{1}, \ldots, u_{s}\right\}$ of $\operatorname{im}(\bar{f})$ to a $k$-basis $\left\{u_{1}, \ldots, u_{n}\right\}$ of $k^{n}=k \otimes_{k[\Gamma]}$ $k[\Gamma]^{n}$. Let $x_{1}, \ldots, x_{n}$ be lifts of the $u_{i}$ to $k[\Gamma]^{n}$ such that $\left\{x_{1}, \ldots, x_{s}\right\} \subset \operatorname{im}(f)$. Since $k[\Gamma]$ is a local ring with the augmentation ideal as the unique maximal ideal ([32], Proposition 7.5.3), Nakayama's lemma implies that $\left\{x_{1}, \ldots, x_{n}\right\}$ generates $k[\Gamma]^{n}$ as a $k[\Gamma]$-module. Since the $k$-dimension of the $k[\Gamma]$-submodule generated by $x_{i}$ is at most $|\Gamma|$ and $\operatorname{dim}_{k} k[\Gamma]^{n}=|\Gamma| \cdot n$, the $k$-dimension of the $k[\Gamma]$-module generated by $\left\{x_{1}, \ldots, x_{i}\right\}$ is $i|\Gamma|$. Because of $\left\{x_{1}, \ldots, x_{s}\right\} \subset \operatorname{im}(f)$,

$$
\operatorname{dim}_{k}(\operatorname{im}(f)) \geq|\Gamma| \cdot s=|\Gamma| \cdot \operatorname{dim}_{k}(\operatorname{im}(\bar{f}))
$$

follows.

Proof of Theorem 1.6. It follows from Lemma 4.1 that, for any finitely presented $k[\Gamma]$-module $M$, the sequence $\left(\operatorname{dim}_{k}\left(k \otimes_{k\left[\Gamma_{i}\right]} M\right) /\left[\Gamma: \Gamma_{i}\right]\right)_{i \geq 0}$ is monotone decreasing. Let $r_{n}$ be the number of $n$-cells in $X$. Let $\partial_{*}$ denote the differentials in the $k[\Gamma]$-complex $C_{*}(\tilde{X} ; k)$. Exactly as in $(2.13)$, one has

$$
\frac{b_{n}\left(X_{i} ; k\right)}{\left[\Gamma: \Gamma_{i}\right]}=\frac{\operatorname{dim}_{k}\left(k \otimes_{k\left[\Gamma_{i}\right]} \operatorname{coker}\left(\partial_{n}\right)\right)}{\left[\Gamma: \Gamma_{i}\right]}+\frac{\operatorname{dim}_{k}\left(k \otimes_{k\left[\Gamma_{i}\right]} \operatorname{coker}\left(\partial_{n+1}\right)\right)}{\left[\Gamma: \Gamma_{i}\right]}-r_{n-1},
$$

from which monotonicity follows. Since Betti numbers are non-negative, the sequence converges.

In the remainder of this section we study the question how we can express the limit

$$
\left(\frac{b_{n}\left(X_{i} ; k\right)}{\left[\Gamma: \Gamma_{i}\right]}\right)_{i \geq 0}
$$

by some algebraic expression in very specific situations. For that we recall the notions of ordered group, the Malcev-Neumann power series ring, and the division closure. 
An ordered group is a group with a strict total ordering of its elements which is invariant under left and right translations. A group which has such an ordering is called orderable. For example, residually torsion-free nilpotent groups are orderable, see [10], Proposition 1.2 on p. 274.

If $\Gamma$ is an ordered group, then the set of formal power series $\sum_{\gamma \in \Gamma} a_{\gamma} \gamma$ with coefficients $a_{\gamma}$ in a skew field $k$ whose support $\left\{\gamma \in \Gamma \mid a_{\gamma} \neq 0\right\}$ is well-ordered becomes a skew field with the obvious ring structure extending the one of the group ring $k[\Gamma]$ (Corollary 15.10 on p. 95 of [9]) which is called the Malcev-Neumann power series ring. We denote it by $k((\Gamma))^{2}$.

Suppose $R$ is a subring of a skew field $K$. Then $D(R, K)$ will denote the division closure of $R$ in $K$, that is the smallest skew subfield of $K$ that contains $R$. If $M_{1}$ and $M_{2}$ are the Malcev-Neumann power series rings of $k[\Gamma]$ with respect to two different orders and $D_{1}, D_{2}$ the division closures of $k[\Gamma]$ in $M_{1}, M_{2}$, respectively, then there is a ring isomorphism $D_{1} \cong D_{2}$ which is the identity on $k[\Gamma]$. This follows from the next theorem. As a consequence, the dimension of the $k((\Gamma))$-vector space

$$
k((\Gamma)) \otimes_{k[\Gamma]} M \cong k((\Gamma)) \otimes_{D(k[\Gamma], k((\Gamma)))}\left(D(k[\Gamma], k((\Gamma))) \otimes_{k[\Gamma]} M\right)
$$

for a $k[\Gamma]$-module $M$ does not depend on the choice of the order on $\Gamma$.

Theorem 4.2. Let $k$ be a skew field, let $\Gamma$ be an orderable group, and let $M_{1}, M_{2}$ be Malcev-Neumann power series rings for $k[\Gamma]$. Set $D_{1}=D\left(k[\Gamma], M_{1}\right)$ and $D_{2}=D\left(k[\Gamma], M_{2}\right)$. Then there is a ring isomorphism $\theta: D_{1} \rightarrow D_{2}$ such that $\theta$ is the identity on $k[\Gamma]$.

Proof. Recall [26], Corollary 1.4, that, being orderable, the group $\Gamma$ is locally indicable, i.e., each finitely generated subgroup $H$ surjects onto $\mathbb{Z}$. Let $J \triangleleft H$ be the kernel of this surjection and pick $t \in H$ so that $H=\langle J, t\rangle$. Let $N_{1}$ and $N_{2}$ denote the subrings of $M_{1}$ and $M_{2}$ respectively consisting of power series with supports in $J$. Then $D\left(k J, D_{1}\right) \subseteq N_{1}$ and $D\left(k J, D_{2}\right) \subseteq N_{2}$. Clearly $\left\{t^{n} \mid n \in \mathbb{N}\right\}$ is linearly independent over $N_{i}$ in $M_{i}$ for $i=1,2$. Theorem 4.2 therefore follows from a slight generalization of Hughes' theorem [11], 7.1 Theorem; all that one needs to do is to modify the proof of 7.2 Lemma in [11]; this is done explicitly in [27], Hughes' Theorem I 6.3.

Theorem 4.3. Let $k$ be a field of characteristic $p>0$. Let $\Gamma$ be a finitely generated group, and let $\Gamma=\Gamma_{0}>\Gamma_{1}>\cdots$ be a descending sequence of normal subgroups such that $\bigcap_{i} \Gamma_{i}=\{1\}$ and each $\Gamma / \Gamma_{i}$ is torsion-free nilpotent. Set $H_{i}=\Gamma_{i} \Gamma^{p^{i}}$ (thus $\Gamma / H_{i}$ is a finite $p$-group). If $X$ is a compact connected $C W$-complex with fundamental group $\Gamma$ and $X_{i}$ the finite cover corresponding to $H_{i}$, then ${ }^{3}$, for every $n$,

$$
\operatorname{dim}_{k((\Gamma))}\left(H_{n}\left(k((\Gamma)) \otimes_{k[\Gamma]} C_{*}(\tilde{X}, k)\right)\right)=\lim _{i \rightarrow \infty} \frac{b_{n}\left(X_{i} ; k\right)}{\left[\Gamma: H_{i}\right]} .
$$

\footnotetext{
${ }^{2}$ We suppress the order in the notation for reasons to be seen below.

${ }^{3}$ Recall that the left-hand side of (4.1) does not depend on the order we choose to define $k((\Gamma))$.
} 
In particular, the limit on the right-hand side is an integer.

Remark 4.4. A large collection of groups are known to be residually torsion-free nilpotent (RTFN): Carl Droms has proved in his PhD thesis [13] (Theorem 1.1 in Chapter III on page 58; see also [1]) that graph groups are RTFN. But this property is inherited by subgroups. It therefore follows from [17] and [4] that free groups, surface groups, reflection groups, right-angled Artin groups and arithmetic hyperbolic groups defined by quadratic forms are virtually RTFN. It follows from the recent breakthrough of Agol [2] and Wise [33] that the fundamental group of a closed hyperbolic 3-manifold is virtually RTFN.We finally note that the proof of Corollary 2.3 in [1] implies that direct and free products of RTFN groups are RTFN.

One deduces the preceding theorem from the following Proposition 4.5 by a similar, even easier, argument as used in the deduction of Theorem 1.1 from Theorem 2.1. More precisely: Similarly as in (2.12) and (2.13) one expresses the left- and righthand side of (4.1) by the cokernels of the $n$-th and $(n+1)$-th differential of the complexes $k((\Gamma)) \otimes_{k[\Gamma]} C_{*}(\tilde{X}, k)$ and $C_{*}\left(X_{i}, k\right)$, respectively; then one applies the proposition below.

Proposition 4.5. Let $k$ be a field of characteristic $p>0$. Let $\Gamma$ be a finitely generated group, and let $\Gamma=\Gamma_{0}>\Gamma_{1}>\cdots$ be a descending sequence of normal subgroups such that $\bigcap_{i} \Gamma_{i}=\{1\}$ and each $\Gamma / \Gamma_{i}$ is torsion-free nilpotent. Set $H_{i}=\Gamma_{i} \Gamma^{p^{i}}$. If $M$ is a finitely presented $k[\Gamma]$-module, then

$$
\operatorname{dim}_{k((\Gamma))}\left(k((\Gamma)) \otimes_{k[\Gamma]} M\right)=\lim _{i \rightarrow \infty} \frac{\operatorname{dim}_{k}\left(k \otimes_{k\left[H_{i}\right]} M\right)}{\left[\Gamma: H_{i}\right]} .
$$

In particular, the limit on the right-hand side is an integer.

A free group is residually torsion-free nilpotent. But even for a free group we cannot say anything for arbitrary residual $p$-chains. In particular, the following question remains open.

Question 4.6. Let $F$ be a finitely generated free group, let $k$ be a field of characteristic $p>0$, and let $F=F_{0}>F_{1}>\cdots$ be a descending sequence of normal subgroups with $F / F_{i}$ a finite $p$-group for all $i$ and $\bigcap_{i \in \mathbb{N}} F_{i}=1$. Let $M$ be a finitely presented $k G$-module. Can $\lim _{i \rightarrow \infty}\left|F / F_{i}\right|^{-1} \operatorname{dim}_{k}\left(k \otimes_{k\left[F_{i}\right]} M\right)$ be transcendental?

In the remainder of this section we are concerned with the proof of Proposition 4.5 for which we need the following lemma.

Lemma 4.7. Let $k$ be an arbitrary skew field, let $M$ be a finitely presented $k[\Gamma]$ module, and let $\Gamma=\Gamma_{0}>\Gamma_{1}>\cdots$ be a descending sequence of normal subgroups with $\Gamma / \Gamma_{i}$ torsion-free nilpotent for all $i$ with $\bigcap_{i \in \mathbb{N}} \Gamma_{i}=1$. Let $D_{i}$ denote the skew 
field of fractions of $k\left[\Gamma / \Gamma_{i}\right]$, which is an Ore localization. Then there exists $m \in \mathbb{N}$ such that

$$
\operatorname{dim}_{D_{i}}\left(D_{i} \otimes_{k[\Gamma]} M\right)=\operatorname{dim}_{k((\Gamma))}\left(k((\Gamma)) \otimes_{k[\Gamma]} M\right) \quad \text { for all } i \geq m
$$

Proof. Choose a nonprincipal ultrafilter $\omega$ on $\mathbb{N}$ and let $D$ denote the ultraproduct of the $D_{i}$ with respect to the ultrafilter $\omega$. Then $D$ is a skew field and $k[\Gamma]$ embeds in $D$. Consider a nontrivial finitely generated subgroup $H$ of $\Gamma$. Let $n$ be the least positive integer such that $H$ is not contained in $\Gamma_{n}$. Then $H / H \cap \Gamma_{n}$ is a nontrivial finitely generated torsion-free nilpotent group, so there exists $N \triangleleft H$ such that $H / N$ is infinite cyclic. Choose $t \in H \backslash N$ so that $H=\langle N, t\rangle$. Then $\left\{t^{i} \mid i \in \mathbb{N}\right\}$ is linearly independent over $k((N))$ and it follows that $\left\{t^{i} \mid i \in \mathbb{N}\right\}$ is linearly independent over $D(k[N], k((\Gamma)))$. Next let $E_{i}$ denote the skew field of fractions of $k\left[N / N \cap \Gamma_{i}\right]$ and form the ultraproduct $E$ of the $E_{i}$ with respect to $\omega$. Then $E$ is a skew field contained in $D$, and $\left\{t^{i} \mid i \in \mathbb{N}\right\}$ is linearly independent over $E$. Therefore $\left\{t^{i} \mid i \in \mathbb{N}\right\}$ is linearly independent over $D(k[N], D)$ and we deduce from [27], Hughes' Theorem I 6.3, that there is an isomorphism $\theta: D(k[\Gamma], k((\Gamma))) \rightarrow D(k[\Gamma], D)$ such that $\theta$ is the identity on $k[\Gamma]$. Therefore $\operatorname{dim}_{k((\Gamma))} k((\Gamma)) \otimes_{k[\Gamma]} M=\operatorname{dim}_{D} D \otimes_{k[\Gamma]} M$. Also $\operatorname{dim}_{D} D \otimes_{k[\Gamma]} M=\lim _{\omega} \operatorname{dim}_{D_{i}} D_{i} \otimes_{k[\Gamma]} M$ and the result follows.

Proof of Proposition 4.5. For each $i \in \mathbb{N}$, let $D_{i}$ denote the skew field of fractions of $k\left[\Gamma / \Gamma_{i}\right]$. By Lemma 4.7, there exists $m_{0} \in \mathbb{N}$ such that $\operatorname{dim}_{D_{m}} D_{m} \otimes_{k[\Gamma]} M=$ $\operatorname{dim}_{k((\Gamma))} k((\Gamma)) \otimes_{k[\Gamma]} M$ for all $m \geq m_{0}$. For $i, m \geq m_{0}$, set $K_{i}^{m}=\Gamma_{m} \Gamma^{p^{i}}$. For any $m \geq m_{0}$ we have

$$
\begin{aligned}
\lim _{i \rightarrow \infty}\left|\Gamma / K_{i}^{m}\right|^{-1} \operatorname{dim}_{k}\left(k \otimes_{k\left[K_{i}^{m}\right]} M\right) & =\operatorname{dim}_{D_{m}}\left(D_{m} \otimes_{k[\Gamma]} M\right) \\
& =\operatorname{dim}_{k((\Gamma))} k((\Gamma)) \otimes_{k[\Gamma]} M
\end{aligned}
$$

by Theorem 6.3 of [23]. The result follows from the monotonicity Lemma 4.1.

\section{Link complements}

Suppose that $\Gamma \rightarrow \mathbb{Z}^{d}$. We embed $\mathbb{Z}^{d} \hookrightarrow \mathbb{Z}_{p}^{d}=: G$ and consider the homomorphism $\phi: \Gamma \rightarrow G$. The $\mathbb{Z}\left[\mathbb{Z}^{d}\right]$-module $H_{1}(\bar{X}, \mathbb{Z})$ is the Alexander invariant of $X$. There is a natural map $\mathbb{Z}\left[\mathbb{Z}^{d}\right] \rightarrow \mathbb{F}_{p}\left[\left[\mathbb{Z}_{p}^{d}\right]\right]$. Since $\mathbb{Z}^{d}$ is amenable, the group ring $\mathbb{F}_{p}\left[\mathbb{Z}^{d}\right]$ is an Ore domain and it follows from [23] that

$$
\begin{aligned}
\beta_{k}\left(\bar{X}, \bar{\Gamma} ; \mathbb{F}_{p}\right) & =\operatorname{rank}_{\mathbb{F}_{p}\left[\left[\mathbb{Z}_{p}^{d}\right]\right]} H_{k}\left(\bar{X}, \mathbb{F}_{p}\left[\left[\mathbb{Z}_{p}^{d}\right]\right]\right) \\
& =\operatorname{rank}_{\mathbb{F}_{p}\left[\mathbb{Z}^{d}\right]} H_{k}\left(\bar{X}, \mathbb{F}_{p}\left[\mathbb{Z}^{d}\right]\right) \\
& =\operatorname{rank}_{\mathbb{F}_{p}\left[t_{1}^{ \pm 1}, \ldots, t_{d}^{ \pm 1}\right]} H_{k}\left(\bar{X}, \mathbb{F}_{p}\left[t_{1}^{ \pm 1}, \ldots, t_{d}^{ \pm 1}\right]\right)
\end{aligned}
$$


This last expression is zero if and only if the (first) Alexander polynomial $\Delta$ of $X$ is non zero modulo $p$.

The above remark in particular applies when $X$ is a 3-manifold with boundary a union of $d$ tori. If $X$ is knot complement (in which case $d=1$ ) the Alexander polynomial $\Delta$ is nonzero and its coefficients are relatively prime. Hence

$$
\beta_{1}\left(\bar{X}, \bar{\Gamma} ; \mathbb{F}_{p}\right)=\operatorname{rank}_{\mathbb{F}_{p}\left[\left[\mathbb{Z}_{p}^{d}\right]\right]} H_{1}\left(\bar{X}, \mathbb{F}_{p}\left[\left[\mathbb{Z}_{p}^{d}\right]\right]\right)=0
$$

and (see also [29], Corollary 4.4)

$$
\lim _{i \rightarrow+\infty} \frac{\operatorname{dim} H_{1}\left(X_{i}, \mathbb{F}_{p}\right)}{\left[\Gamma: \Gamma_{i}\right]}=0 .
$$

In that case one may even deduce from (5.1) that $H_{1}\left(X_{i}, \mathbb{F}_{p}\right)=\mathbb{F}_{p}$ for all $i$, see Lemma 5.4 of [6].

We may as well consider the case of a link complement $l=l_{1} \cup \cdots \cup l_{d}$ in $\mathbb{S}^{3}$. Recall that, in the case $d=2, \Delta(1)$ is equal to the linking number $\operatorname{Lk}\left(l_{1}, l_{2}\right)$ of the two components of the link. The same proof as in the knot case then shows that if $p$ is a prime that does not divide $\operatorname{Lk}\left(l_{1}, l_{2}\right)$, then

$$
\lim _{i \rightarrow+\infty} \frac{\operatorname{dim} H_{1}\left(X_{i}, \mathbb{F}_{p}\right)}{\left[\Gamma: \Gamma_{i}\right]}=0 .
$$

One may wonder if the proof may be extended to show that $H_{1}\left(X_{i}, \mathbb{F}_{p}\right)=\mathbb{F}_{p}^{2}$ for all $i$ as is true according to [29], Theorem 5.11.

We conclude this note by the proof of Proposition 1.5.

Proof of Proposition 1.5. It follows from [20] that there exists a link $l$ with 2 components such that $\Delta(t, t)=p$. Now note that $\Delta(t, t)$ is the Alexander polynomial associated to the abelian cover of $X$ corresponding to the map $\Gamma \rightarrow \mathbb{Z}^{2} \rightarrow \mathbb{Z}^{2} /\langle a-b\rangle \cong \mathbb{Z}$. Since $\Delta(t, t)$ is non zero,

$$
\lim _{i \rightarrow+\infty} \frac{\operatorname{dim} H_{1}\left(X_{i}, \mathbb{Q}\right)}{\left[\Gamma: \Gamma_{i}\right]}=0 .
$$

But since $\Delta(t, t)$ is zero modulo $p$, we have

$$
\lim _{i \rightarrow+\infty} \frac{\operatorname{dim} H_{1}\left(X_{i}, \mathbb{F}_{p}\right)}{\left[\Gamma: \Gamma_{i}\right]} \neq 0 .
$$

Other examples of closed finite $C W$-complexes with a chain

$$
\pi_{1}(X)=\Gamma_{0} \supseteq \Gamma_{1} \supseteq \Gamma_{2} \supseteq \cdots
$$

of normal subgroups of finite index in $\pi_{1}(X)$ such that $\lim _{i \rightarrow+\infty} \frac{\operatorname{dim} H_{1}\left(X_{i}, \mathbb{F}_{p}\right)}{\left[\Gamma: \Gamma_{i}\right]} \neq$ $\lim _{i \rightarrow+\infty} \frac{\operatorname{dim} H_{1}\left(X_{i}, \mathbb{Q}\right)}{\left[\Gamma: \Gamma_{i}\right]}$ holds can be found in [15] and [25]. One can additionally arrange $X=B \Gamma$ or $\bigcap_{i \geq 0} \Gamma_{i}=\{1\}$. However, the problem is still open to find an example with both $X=B \Gamma$ and $\bigcap_{i \geq 0} \Gamma_{i}=\{1\}$. 


\section{References}

[1] I. Agol, Criteria for virtual fibering. J. Topol. 1 (2008), no. 2, 269-284. Zbl 1148.57023 MR 2399130

[2] I. Agol, The virtual Haken conjecture. With an appendix by Agol, Daniel Groves, and Jason Manning, Doc. Math. 18 (2013), 1045-1087. Zbl 1286.57019 MR 31045

[3] K. Ardakov and K. A. Brown, Ring-theoretic properties of Iwasawa algebras: a survey. Doc. Math. Extra Vol. (2006), 7-33. Zbl 1125.16011 MR 2290583

[4] N. Bergeron, F. Haglund, and D. T. Wise, Hyperplane sections in arithmetic hyperbolic manifolds. J. London Math. Soc. (2) 83 (2011), no. 2, 431-448. Zbl 1236.57021 MR 2776645

[5] F. Calegari and M. Emerton, Bounds for multiplicities of unitary representations of cohomological type in spaces of cusp forms. Ann. of Math. (2) 170 (2009), no. 3, 1437-1446. Zbl 1195.22015 MR 2600878

[6] F. Calegari and M. Emerton, Mod- $p$ cohomology growth in $p$-adic analytic towers of 3-manifolds. Groups Geom. Dyn. 5(2011), no. 2, 355-366. Zbl 1242.57014 MR 2782177

[7] F. Calegari and M. Emerton, Completed cohomology - a survey. In Non-abelian fundamental groups and Iwasawa theory, London Math. Soc. Lecture Note Ser. 393, Cambridge University Press, Cambridge 2012, 239-257. Zbl 06059400 MR 2905536

[8] B. Clair and K. Whyte, Growth of Betti numbers. Topology 42 (2003), no. 5, 1125-1142. Zbl 1031.58021 MR 1978050

[9] P. M. Cohn, Free ideal rings and localization in general rings. New Math. Monogr. 3, Cambridge University Press, Cambridge 2006. Zbl 1114.16001 MR 2246388

[10] P. Dehornoy, I. Dynnikov, D. Rolfsen, and B. Wiest, Ordering braids. Math. Surveys Monogr. 148, Amer. Math. Soc., Providence, RI, 2008. Zbl 1163.20024 MR 2463428

[11] W. Dicks, D. Herbera, and J. Sánchez, On a theorem of Ian Hughes about division rings of fractions. Comm. Algebra 32, (2004), no. 3, 1127-1149. Zbl 1084.16018 MR 2063801

[12] J. D. Dixon, M. P. F. du Sautoy, A. Mann, and D. Segal, Analytic pro-p groups. Second edition, Cambridge Stud. Adv. Math. 61, Cambridge University Press, Cambridge 1999. Zbl 0934.20001 MR 1720368

[13] C. Droms, Graph groups. PhD thesis, Syracuse University, 1983.

[14] M. Emerton, On the interpolation of systems of eigenvalues attached to automorphic Hecke eigenforms. Invent. Math. 164 (2006), no. 1, 1-84. Zbl 1090.22008 MR 2207783

[15] M. Ershov, W. Lück, and D. Osin, The first $l^{2}$-Betti number and approximation in prime characteristics. In preparation.

[16] D. R. Farkas and P. A. Linnell, Congruence subgroups and the Atiyah conjecture. In Groups, rings and algebras, Contemp. Math. 420, Amer. Math. Soc., Providence, RI, 2006, 89-102. Zbl 1131.16013 MR 2279234

[17] F. Haglund and D. T. Wise, Special cube complexes. Geom. Funct. Anal. 17 (2008), no. 5, 1551-1620. Zbl 1155.53025 MR 2377497

[18] M. Harris, $p$-adic representations arising from descent on abelian varieties. Compositio Math. 39 (1979), no. 2, 177-245. Zbl 0417.14034 MR 0546966 
[19] M. Harris, Correction to: " $p$-adic representations arising from descent on abelian varieties" [Compositio Math. 39 (1979), no. 2, 177-245]. Compositio Math. 121 (2000), no. 1, 105-108. Zbl 0417.14034 MR 1753112

[20] F. Hosokawa, On $\nabla$-polynomials of links. Osaka Math. J. 10 (1958), 273-282. Zbl 0105.17404 MR 0102820

[21] M. Lackenby, Large groups, property $(\tau)$ and the homology growth of subgroups. Math. Proc. Cambridge Philos. Soc. 146 (2009), no. 3, 625-648. Zbl 1185.57014 MR 2496348

[22] M. Lackenby, Detecting large groups. J. Algebra 324 (2010), no. 10, 2636-2657. Zbl 1231.20026 MR 2725193

[23] P. Linnell, W. Lück, and R. Sauer, The limit of $\mathbb{F}_{p}$-Betti numbers of a tower of finite covers with amenable fundamental groups. Proc. Amer. Math. Soc. 139 (2011), no. 2, 421-434. Zbl 1272.55006 MR 2736326

[24] W. Lück, Approximating $L^{2}$-invariants by their finite-dimensional analogues. Geom. Funct. Anal. 4 (1994), no. 4, 455-481. Zbl 0853.57021 MR 1280122

[25] W. Lück, Approximating $L^{2}$-invariants and homology growth. Geom. Funct. Anal. 23 (2013), no. 2, 622-663. Zbl 1273.22009 MR 3053758

[26] A. Rhemtulla and D. Rolfsen, Local indicability in ordered groups: braids and elementary amenable groups. Proc. Amer. Math. Soc. 130 (2002), no. 9, 2569-2577. Zbl 0996.20024 MR 1900863

[27] J. Sánchez, Localization: on division rings and tilting modules. PhD thesis, Universitat Autònoma de Barcelona, 2008.

[28] P. Sarnak and X. X. Xue, Bounds for multiplicities of automorphic representations. Duke Math. J. 64 (1991), no. 1, 207-227. Zbl 0741.22010 MR 1131400

[29] D. S. Silver and S. G. Williams, Torsion numbers of augmented groups with applications to knots and links. Enseign. Math. (2) 48 (2002), no. 3-4, 317-343. Zbl 1037.57004 MR 1955605

[30] L. Wall, Homology in finite index subgroups. PhD Thesis, University of Oxford, 2009, available at www.maths.ox.ac.uk/ lackenby/foo.pdf.

[31] C. A. Weibel, An introduction to homological algebra. Cambridge Stud. Adv. Math. 38, Cambridge University Press, Cambridge 1994. Zbl 0797.18001 MR 1269324

[32] J. S. Wilson, Profinite groups. London Math. Soc. Monogr. (N.S.) 19, The Clarendon Press; Oxford University Press, New York 1998. Zbl 0909.20001 MR 1691054

[33] D. T. Wise, The structure of groups with a quasiconvex hierarchy. Preprint, 2009. www.math.mcgill.ca/wise/papers.htm

Received September 28, 2012; revised January 27, 2013

N. Bergeron, Institut de Mathématiques de Jussieu, Unité Mixte de Recherche 7586 du CNRS, Université Pierre et Marie Curie, 4, place Jussieu, 75252 Paris Cedex 05, France E-mail: bergeron@math.jussieu.fr

P. Linnell, Department of Mathematics, Virginia Tech, Blacksburg, VA 24061-0123, U.S.A.

E-mail: plinnell@math.vt.edu 
W. Lück, Mathematisches Institut der Universität Bonn, Endenicher Allee 60, 53115 Bonn, Germany

E-mail: wolfgang.lueck@him.uni-bonn.de

R. Sauer, Institute of Algebra and Geometry, Karlsruhe Institute of Technology, 76133 Karlsruhe, Germany

E-mail: roman.sauer@kit.edu 